\title{
Three-minute constant rate step test for detecting exertional dyspnea relief after bronchodilation in COPD
}

This article was published in the following Dove Press journal: International Journal of COPD

29 November 2016

Number of times this article has been viewed

\section{Benoit Borel ${ }^{1,2}$ Maitland ${ }^{3}$ \\ Alan Hamilton ${ }^{4}$ \\ Jean Bourbeau ${ }^{5}$ \\ Hélène Perrault ${ }^{6}$ \\ Dennis Jensen ${ }^{3,5,7}$ \\ François Maltais ${ }^{2}$}

Courtney A Wilkinson-

'Laboratoire HAVAE, Université de Limoges, Limoges, France; ${ }^{2}$ Centre de Recherche, Institut Universitaire de Cardiologie et de Pneumologie de Québec, Université Laval, Québec, ${ }^{3}$ Clinical Exercise and Respiratory Physiology Laboratory, Department of Kinesiology and Physical Education, McGill University, Montréal, QC, ${ }^{4}$ Boehringer Ingelheim (Canada) Limited, Burlington, ON, ${ }^{5}$ Respiratory Epidemiology and Clinical Research Unit, Montreal Chest Institute, McGill University Health Center, Montreal, QC, ${ }^{6}$ Faculty of Health Sciences, University of Ottawa, Ottawa, ON, ${ }^{7}$ Translational Research in Respiratory Diseases Program, Research Institute of the McGill University Health Centre, Montreal, QC, Canada
Correspondence: François Maltais Centre de Pneumologie, Institut Universitaire de Cardiologie et de Pneumologie de Québec, 2725 Chemin Sainte-Foy, Québec, QC GIV 4G5, Canada

Fax +I 4186564762

Email francois.maltais@fmed.ulaval.ca
Background: The aim of this study was to evaluate the responsiveness of the 3-minute constant rate step test (3-MST) to detect the relief of exertional dyspnea (respiratory discomfort) after acute bronchodilation in COPD patients.

Patients and methods: A total of 40 patients with moderate-to-severe COPD (mean forced expiratory volume in 1 second: $45.7( \pm 14.7), \%$ predicted) performed four 3-MSTs at randomly assigned stepping rates of 14, 16, 20 and 24 steps/min after inhalation of nebulized ipratropium bromide $(500 \mu \mathrm{g}) / \mathrm{salbutamol}(2.5 \mathrm{mg})$ and saline placebo, which were randomized to order. Patients rated their intensity of perceived dyspnea at the end of each 3-MST using Borg 0-10 category ratio scale.

Results: A total of 37 (92.5\%), $36(90 \%), 34$ (85\%) and 27 (67.5\%) patients completed all 3 minutes of exercise at 14,16, 20 and 24 steps/min under both treatment conditions, respectively. Compared with placebo, ipratropium bromide/salbutamol significantly decreased dyspnea at the end of the third minute of exercise at 14 steps $/$ min (by $0.6 \pm 1.0$ Borg $0-10$ scale units, $P<0.01$ ) and 16 steps $/ \min$ (by $0.7 \pm 1.3$ Borg $0-10$ scale units, $P<0.01$ ); however, no statically significant differences were observed between treatments at 20 and 24 steps $/ \mathrm{min}$ (both $P>0.05$ ).

Conclusion: The 3-MST, when performed at 14 and 16 steps/min, was responsive to detect the relief of exertional dyspnea after acute bronchodilation in patients with moderate-to-severe COPD.

Keywords: chronic obstructive pulmonary disease, exercise testing, dyspnea, intervention

\section{Introduction}

Exertional dyspnea is the primary symptomatic manifestation reported in COPD, ${ }^{1}$ and it dominates the clinical presentation of this disease. The evaluation of dyspnea and exercise tolerance is thus critical to the clinical management of COPD, particularly as it relates to assessing the efficacy of therapeutic interventions. Indeed, therapeutic decisions and treatment effectiveness should be based, in large part, on the assessment of patient-reported changes in physical activity-related dyspnea., ${ }^{2,3}$

Although walking and cycling exercise protocols are currently available for the evaluation of exercise tolerance, ${ }^{4}$ none of these tests are perfectly designed to evaluate the impact of therapeutic interventions on exertional dyspnea as the primary end point. Constant work-rate cycling protocols and the endurance shuttle walking test are typically conducted up to symptom limitation with the intention of quantifying exercise duration. However, the open nature of these tests makes it difficult to evaluate the effects of therapy on end-exercise dyspnea. One strategy to address this difficulty is to compare dyspnea ratings at a standardized submaximal time (iso-time) during 
exercise while controlling the walking speed during an endurance shuttle walking test, ${ }^{5}$ the power output during a constant rate cycling test $\mathrm{t}^{6-8}$ or the inclination and belt speed during a constant rate treadmill test. ${ }^{9,10}$ One potential limitation with this approach is that pre- and post-intervention dyspnea measurements are not always obtained at the same time point since the duration of the exercise is variable. In addition, interindividual comparisons of the magnitude of treatment effect are difficult to make due to variations in iso-time from one patient to another.

Self-paced walking or stair climbing tests are some of the most commonly used field tests in COPD. ${ }^{4}$ These tests are most appropriate for the evaluation of exercise tolerance (endurance) in COPD but are not optimally designed to evaluate the effects of therapies on exertional dyspnea, due to lack of control of the exercise stimulus.

To circumvent these limitations in the assessment of exertional dyspnea, Perrault et al ${ }^{11}$ validated two simple field-based exercise tests that were specifically developed to assess the impact of therapeutic interventions on exertional dyspnea: the 3-minute constant rate step test (3-MST) and the 3-minute constant rate shuttle walking test. By externally controlling the walking speed/stepping rate and limiting the duration of exercise to 3 minutes, these tests provoke a measureable and reproducible level of dyspnea (at a standardized and reproducible exercise stimulus) that may be encountered by patients with COPD during their activities of daily living ${ }^{12-14}$ and that may be amenable to therapy. ${ }^{11}$ To this end, a randomized, double-blind, placebo-controlled, crossover study from our group recently confirmed the responsiveness of the 3-minute constant rate shuttle walking test to detect statistically significant and clinically meaningful relief of exertional dyspnea after acute bronchodilation in patients with moderate-to-severe COPD. ${ }^{15}$ The aim of the current study was to assess the responsiveness of the 3-MST to detect the bronchodilator-induced relief of exertional dyspnea in COPD, testing the hypothesis that this exercise test is responsive to detect statistically significant and clinically meaningful relief of exertional dyspnea after acute bronchodilation in patients with moderate-to-severe COPD.

\section{Patients and methods}

\section{Study population}

Patients with COPD were recruited from two different centers in Québec, Canada (Institut Universitaire de Cardiologie et de Pneumologie de Québec [IUCPQ] and the Montreal Chest Institute [MCI]), and a total of 40 ambulatory and clinically stable men and women were included. The following eligibility criteria were used: age $>50$ years, smoking history $>10$ pack-years, post-bronchodilator forced expiratory volume in 1 second $\left(\mathrm{FEV}_{1}\right)$ between $30 \%$ and $79 \%$ predicted and $\mathrm{FEV}_{1} /$ forced vital capacity (FVC) $<70 \%$ (Global Initiative for Chronic Obstructive Lung Disease [GOLD] stage 2 and 3). ${ }^{3}$ Potential participants were excluded in the presence of a respiratory exacerbation within the preceding 2 months, history of asthma, oxygen pulse saturation $\left(\mathrm{SpO}_{2}\right)<85 \%$ at rest or during exercise or in the presence of clinically evident cardiovascular, neurological, orthopedic, hematological diseases that could influence exercise tolerance or may prevent the realization of the step test.

\section{Study design}

As illustrated in Figure 1, this was a randomized, doubleblind, placebo-controlled, crossover, two-site study (ClinicalTrials.gov, registration number: NCT01655199), wherein eligible patients visited the research facility four times, with a period of 3-7 days within visits. The study protocol and consent form were approved by each institution's Biomedical Research Ethics Board in accordance with the Declaration of Helsinki (IUCPQ, \#CER20819;

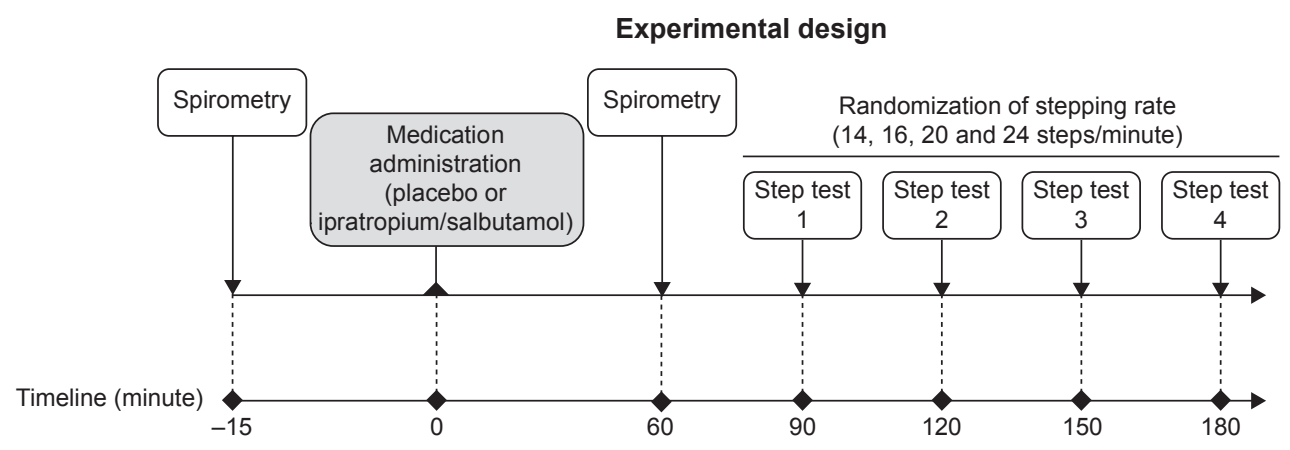

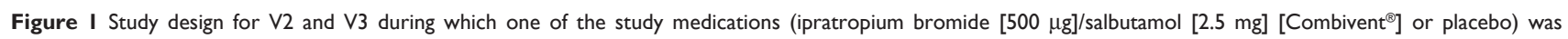
administered.

Abbreviations: V2, visit 2; V3, visit 3 . 
MCI, 12-327-BMA). Written informed consent and medical history were obtained at visit 0 (V0), and inhaled medication was modified, if necessary (details are given in the following section). During visit 1 (V1), complete pre- and post-bronchodilator ( $200 \mu \mathrm{g}$ salbutamol) pulmonary function testing was performed, followed by a symptom-limited peak incremental cycle exercise test and then a 3-MST for familiarization purposes. Visits 2 and 3 (V2, V3) were identical with the exception of the nebulized medication that was used: ipratropium bromide $(500 \mu \mathrm{g}) /$ salbutamol (2.5 mg; Combivent ${ }^{\circledR}$; Boehringer Ingelheim (Canada) Ltd., Mississauga, ON, Canada) or $0.9 \%$ saline placebo (same volume of nebulized saline solution) whose order was randomized. V2 and V3 included pre-dose and 1-hour post-dose spirometry followed immediately by four 3-MSTs (interspersed by 30 minutes of rest) at randomly assigned stepping rates of 14, 16, 20 and $24 \mathrm{steps} / \mathrm{min}$. The 3-MSTs were administered at 30 -minute intervals to provide sufficient recovery time to study participants while ensuring that all tests could be completed during maximum bronchodilation effects of study medication. ${ }^{16}$ A research pharmacist at both sites who was unblinded to study medication, but not otherwise involved in the study, performed treatment randomization according to a computer-generated randomization list and was responsible for blinding and dispensing of study medications.

\section{Pulmonary function testing}

Standard pulmonary function tests, including routine spirometry, constant volume body plethysmography and single-breath determination of diffusing capacity of the lung for carbon monoxide $\left(\mathrm{D}_{\mathrm{L}} \mathrm{CO}\right)$, were conducted according to established guidelines ${ }^{17}$ using automated equipment and referenced to predicted normal values. ${ }^{18,19}$ Maximum voluntary ventilation was estimated by multiplying $\mathrm{FEV}_{1}$ by $35 .{ }^{20}$

\section{Concomitant therapeutic interventions}

With the exception of tiotropium, the only long-acting anticholinergic agent available in Canada at the time of this study, usual respiratory medications were maintained throughout the study. Because of its long washout period, tiotropium was switched to ipratropium bromide (Atrovent ${ }^{\circledR}$; Boehringer Ingelheim (Canada) Ltd.) four times daily starting from V0 and for a period of $\geq 2$ weeks prior to V1. Before V2 and V3, patients withdrew from short- and long-acting $\beta_{2}$-agonists (6 and 24 hours), short-acting anticholinergics (6 hours) and theophyllines (48 hours). If any short-acting bronchodilator was taken as rescue medication within 6 hours of V1, V2 or V3, the visit was rescheduled.

\section{Exercise tests}

\section{Incremental exercise test}

Incremental exercise tests were performed on an electronically braked cycle ergometer (Lode Corival, Lode BV, Groningen, the Netherlands; MCI, Ergoline 800s; Viasys Healthcare, Bitz, Germany) and consisted of a rest period of $\geq 5$ minutes, followed by $10 \mathrm{~W} / \mathrm{min}$ increases in power output (starting at $10 \mathrm{~W}$ ) to symptom limitation. Standard cardiopulmonary parameters were collected breath by breath, using automated equipment (Vmax Spectra ${ }^{\mathrm{TM}}$ [IUCPQ] and Vmax Encore ${ }^{\mathrm{TM}}$ [MCI], Sensormedics; CareFusion, Yorba Linda, CA, USA) at rest and during cycle exercise. Inspiratory capacity (IC) maneuvers ${ }^{21}$ and Borg 0-10 scale $^{22}$ intensity ratings of perceived dyspnea and leg discomfort were collected at rest and at end exercise. Inspiratory reserve volume (IRV) was calculated as the difference between IC and the simultaneously determined tidal volume $\left(V_{\mathrm{T}}\right)$. Peak power output was defined as the highest power output that the patient was able to sustain for $\geq 30$ seconds. Symptom-limited peak values of oxygen uptake $\left(\dot{V} \mathrm{O}_{\text {2peak }}\right)$, carbon dioxide production $\left(\dot{V} \mathrm{CO}_{2}\right)$, peak ventilation $\left(\dot{V}_{\text {Epeak }}\right), V_{\mathrm{T}}$, breathing frequency $(f)$, peak heart rate (HR) and $\mathrm{SpO}_{2}$ were taken as the average of the last 30 seconds of loaded pedaling. $\mathrm{SpO}_{2}$ and $\mathrm{HR}$ were monitored by finger pulse oximetry (IUCPQ, Nellcor N-395; Soma Technology, Bloomfield, CT, USA; MCI, Ohmeda Biox 3740; Louisville, CO, USA) and 12-lead electrocardiogram (CardioSoft; CareFusion), respectively. Finally, peak values for power output, $\dot{V} \mathrm{O}_{2 \text { peak }}$ and HR were referenced to their respective predicted normal values. ${ }^{23}$

\section{3-MST}

The 3-MST protocol consisted of four randomly assigned 3-minue bouts of exercise (interspersed by 30 minutes of rest) at externally paced stepping rates of 14, 16, 20 and 24 steps/min. As described by Perrault et al, ${ }^{11}$ patients started stepping on hearing the audio instructions for "step-up" indicating to place both feet up onto the first stair of the step one after the other ( $20 \mathrm{~cm}$ rise; $22 \mathrm{~cm}$ depth), with the rate being targeted to the movement of each foot and "step-down" indicating to step back down to the floor, one foot after the other. Patients were instructed to match their step rate with the audio signal for the entire 3-minute bout of the exercise or until they became symptom limited (ie, they were unable to keep pace with the audio signals due to intolerable symptoms), whichever occurred first.

Measures of $\dot{V} \mathrm{O}_{2}, \dot{V} \mathrm{CO}_{2}, \dot{V}_{\mathrm{E}}, V$ T, $f$ R and $\mathrm{SpO}_{2}$ were collected breath by breath at rest and during each of the 3-MSTs, using a Jaeger Oxycon Mobile ${ }^{\circledR}$ system (CareFusion, Hoechberg, Germany) at the IUCPQ and a Vmax Encore ${ }^{\mathrm{TM}}$ 
cardiopulmonary exercise testing system (Sensormedics) at the MCI. Using the Borg 0-10 scale, patients rated the intensity of their perceived dyspnea and leg discomfort at rest, prior to the start of each 3-MST, and within the last 30 seconds of each 3-MST or at the symptom-limited peak of each 3-MST, whichever occurred first. IC maneuvers were performed at rest prior to the start of each 3-MST and immediately after symptom ratings at 3 minutes or at the symptom-limited peak of each 3-MST. ${ }^{21}$ Physiological parameters were averaged over the last 30 seconds of the third minute of each 3-MST (or over the last 30 seconds of exercise if the patient stopped due to intolerable symptoms prior to completing the third minute of a given 3-MST) for each patient and linked with the corresponding symptom ratings and IC-derived measurements.

\section{Statistical analysis}

No pilot data were available to support a formal sample size calculation. The sample size was thus estimated based on the previous work of our research group showing that 40 patients were sufficient to demonstrate the responsiveness of the 3-minute constant rate shuttle walking test to detect the improvement in exertional dyspnea following bronchodilation. ${ }^{15}$ Based on this past experience, we estimated that a similar number of patients should be enrolled in the current study. The effects of study medications (ipratropium bromide/salbutamol vs placebo) and measurement time (pre- vs post-dose) on $\mathrm{FEV}_{1}, \mathrm{FVC}$ and $\mathrm{FEV}_{1}$ / FVC were examined using a two-way repeated measures analysis of variance with correction for multiple comparisons using Bonferroni's post hoc tests (Prism 6; GraphPad Software, La Jolla, CA, USA). The changes in Borg 0-10 scale intensity ratings of dyspnea (primary outcome variable) and in each of the secondary outcome variables (ie, Borg 0-10 scale intensity ratings of leg discomfort, $\dot{V} \mathrm{O}_{2}$, $\dot{V} \mathrm{CO}_{2}, \dot{V}_{\mathrm{E}}, V_{\mathrm{T}}, f, \mathrm{HR}, \mathrm{SpO}_{2}, \mathrm{IC}$ and $\mathrm{IRV}$ ) recorded at the end of the third minute of each of the four 3-MSTs after inhalation of nebulized ipratropium bromide/salbutamol vs placebo were analyzed with two-tailed paired Student's $t$-tests. For the exercise tests, where a patient was unable to complete the entire 3 minutes of exercise, a missing value was considered for 3-minute Borg dyspnea and leg discomfort scores, and values at iso-time (corresponding to the longest exercise time completed in both conditions) were considered for cardiorespiratory parameters. The proportion of patients reaching a 1-point difference in Borg dyspnea intensity ratings, the accepted minimal clinically important difference for this variable, ${ }^{24}$ was calculated.
Statistical significance was set at $P \leq 0.05$, and the results are expressed as mean $\pm \mathrm{SD}$.

\section{Results \\ Study participants}

A flowchart of the study is presented in Figure 2.

A total of 40 patients with GOLD stage 2 and 3 COPD were recruited between January 2012 and June 2014. Baseline data of the study population are provided in Table 1. On average, patients had static lung hyperinflation, pulmonary gas trapping and low diffusion capacity $\left(\mathrm{D}_{\mathrm{L}} \mathrm{CO}\right)$. At symptom-limited, peak incremental cycle exercise, patients reported "severe" to "very severe" dyspnea and leg discomfort and exhibited low peak power output, $\dot{V} \mathrm{O}_{2 \text { peak }}$ and HR in association with marked dynamic lung hyperinflation (as evidenced by a $0.70 \mathrm{~L}$ IC decrease from rest to peak exercise), ventilatory inefficiency and complete or near-complete erosion of ventilatory reserve and IRV (Table 2).

\section{Effects of study medication on pulmonary function}

In contrast to placebo, ipratropium bromide/salbutamol significantly increased $\mathrm{FEV}_{1}, \mathrm{FVC}$ and $\mathrm{FEV}_{1} / \mathrm{FVC}$ by an average of $\sim 20 \%, \sim 14 \%$ and $\sim 7 \%$ above their pre-dose values, respectively (Table 3 ).

\section{Dyspnea responses during the 3-MST}

Resting Borg dyspnea scores ranged from 0.31 to 0.44 ("very very slight" to "very slight"). A total of 37 (93\%), 36 (90\%), $34(85 \%)$ and $27(68 \%)$ patients were able to complete all 3 minutes of exercise at 14, 16, 20 and 24 steps/min under both treatment conditions, respectively. Compared with placebo, ipratropium bromide/salbutamol significantly decreased intensity ratings of dyspnea at the end of the third minute of exercise at $14 \mathrm{steps} / \mathrm{min}$ (by $0.6 \pm 1.0$ Borg $0-10$ scale unit) and 16 steps/min (by $0.7 \pm 1.3$ Borg 0-10 scale unit); however, no such between-treatment significant differences were seen at 20 and 24 steps/min (Figure 3 ).

Individual patient's post-dose changes in Borg 0-10 scale intensity ratings of dyspnea are presented in the Figure S1: after treatment with ipratropium bromide/salbutamol vs placebo, dyspnea intensity ratings decreased by $\geq 1$ Borg $0-10$ scale units at the end of the third minute of exercise in $18(45 \%), 18(45 \%), 13(32.5 \%)$ and $11(27.5 \%)$ patients at 14, 16, 20 and 24 steps/min, respectively (Supplementary

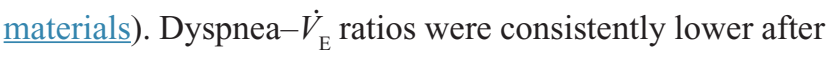
inhalation of ipratropium bromide/salbutamol compared with placebo (Table 4 and Figure S2). In contrast, the reduction in 


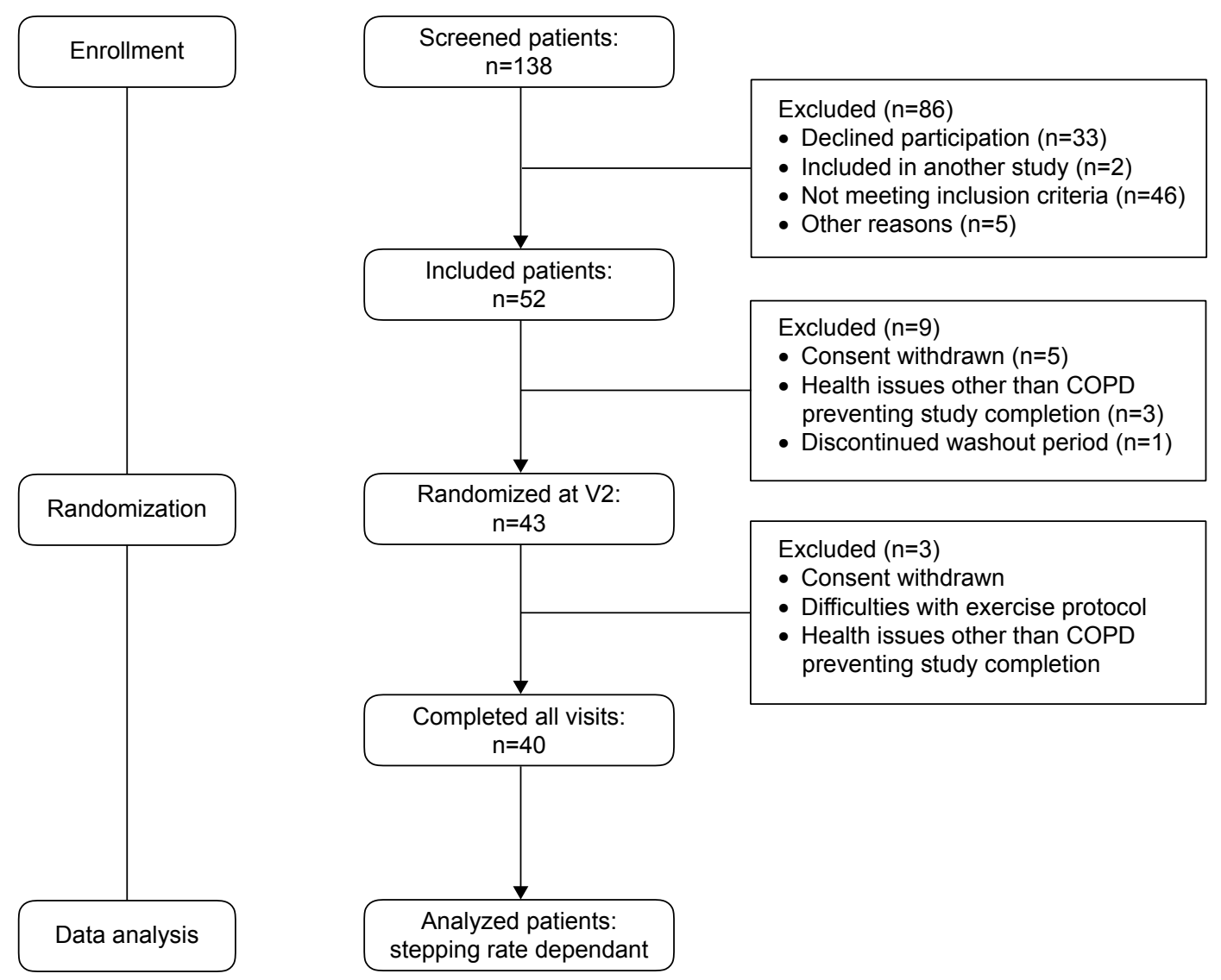

Figure 2 Flowchart of the two-site clinical study. Abbreviation: $\mathrm{V} 2$, visit 2.

Table I Baseline characteristics of the study population $(n=40)$

\begin{tabular}{|c|c|}
\hline Parameter & Mean (SD) \\
\hline Male/female & $33 / 7$ \\
\hline Age (years) & $69(7)$ \\
\hline Height (cm) & $168(8)$ \\
\hline Weight (kg) & $74(15)$ \\
\hline Body mass index $\left(\mathrm{kg} \cdot \mathrm{m}^{-2}\right)$ & $25.9(4.5)$ \\
\hline Smoking history (pack-years) & $57(34)$ \\
\hline \multicolumn{2}{|l|}{ Pulmonary function } \\
\hline \multicolumn{2}{|l|}{ COPD severity (GOLD³), n (\%) } \\
\hline Moderate & $18(45)$ \\
\hline Severe & $22(55)$ \\
\hline Pre-BD FEV $($ (L); \% predicted & $1.24(0.41) ; 46(14)$ \\
\hline Post-BD FEV $(\mathrm{L}) ; \%$ predicted & I.49 (0.44); 55 (I5) \\
\hline $\mathrm{FVC}(\mathrm{L})$ & $2.97(0.90)$ \\
\hline FVC (\% predicted) & $82(21)$ \\
\hline $\mathrm{FEV}_{\mathrm{I}} / \mathrm{FVC}(\%)$ & $43(14)$ \\
\hline FRC (\% predicted) & $138(33)$ \\
\hline TLC (\% predicted) & $112(16)$ \\
\hline RV (\% predicted) & $152(45)$ \\
\hline $\mathrm{D}_{\mathrm{L}} \mathrm{CO}$ (\% predicted) & $77(22)$ \\
\hline
\end{tabular}

Abbreviations: $B D$, bronchodilator; $D_{L} C O$, diffusing capacity of the lung for carbon monoxide; $\mathrm{FEV}_{1}$, forced expiratory volume in I second; $F R C$, forced residual capacity; FVC, forced vital capacity; GOLD, Global Initiative for Chronic Obstructive Lung Disease; RV, residual volume; TLC, total lung capacity.
Table 2 Physiological and perceptual responses at the symptomlimited peak incremental cycle exercise $(n=40)$

\begin{tabular}{|c|c|}
\hline Parameter & Mean (SD) \\
\hline Power output (W) & $76(29)$ \\
\hline Power output (\% predicted) & $55.8(18.3)$ \\
\hline$\dot{\mathrm{V}} \mathrm{O}_{2}\left(\mathrm{~mL} \cdot \mathrm{kg}^{-1} \cdot \mathrm{min}^{-1}\right)$ & $18.4(4.7)$ \\
\hline$\dot{\mathrm{V}} \mathrm{O}_{2}(\%$ predicted $)$ & $82.4(17.9)$ \\
\hline$\dot{\mathrm{V}} \mathrm{CO}_{2}\left(\mathrm{~mL} \cdot \mathrm{kg}^{-1} \cdot \mathrm{min}^{-1}\right)$ & $18.9(6.0)$ \\
\hline$\dot{V}_{\mathrm{E}} / \dot{\mathrm{V}} \mathrm{CO}_{2}$ & $36.8(6.6)$ \\
\hline HR (beats $\cdot \min ^{-1}$ ) & $|2|(2 \mid)$ \\
\hline HR (\% predicted) & $72.8(12.0)$ \\
\hline $\mathrm{SpO}_{2}(\%)$ & $94.1(2.7)$ \\
\hline$\dot{V}_{E}\left(L \cdot \min ^{-1}\right)$ & $48.9(14.1)$ \\
\hline$\dot{V}_{\mathrm{E}}(\% \mathrm{MVV})$ & $99(17)$ \\
\hline$V_{\mathrm{T}}(\mathrm{L})$ & $1.39(0.27)$ \\
\hline$f$ (breaths $\left.\cdot \mathrm{min}^{-1}\right)$ & $35.8(6.34)$ \\
\hline IC (L) & $\mathrm{I} .89(0.48)$ \\
\hline$\Delta \mathrm{IC}$ rest-peak exercise $(\mathrm{L})$ & $-0.70(0.36)$ \\
\hline IRV (L) & $0.48(0.29)$ \\
\hline Dyspnea (Borg 0-10 scale units) & $6.2(2.0)$ \\
\hline Leg discomfort (Borg 0-10 scale units) & $6.3(2.1)$ \\
\hline
\end{tabular}

Abbreviations: $f$, breathing frequency; $\Delta$, change from rest to end exercise; $\mathrm{SpO}_{2}$, oxygen pulse saturation; $\dot{V} \mathrm{CO}_{2}, \mathrm{CO}_{2}$ production; $\dot{V}_{\mathrm{E}}$, ventilation; $\dot{V}_{\mathrm{E}} / \dot{V}_{\mathrm{CO}}$, ventilatory equivalent for $\mathrm{CO}_{2} ; \mathrm{VO}_{2}, \mathrm{O}_{2}$ uptake; $V_{\mathrm{T}}$, tidal volume. $\mathrm{HR}$, heart rate; IC, inspiratory capacity; IRV, inspiratory reserve volume; MVV, maximum voluntary ventilation. 
Table 3 Effects of nebulized ipratropium bromide $(500 \mu \mathrm{g}) /$ salbutamol $(2.5 \mathrm{mg})$, and $0.9 \%$ saline placebo on spirometry parameters at rest $(n=40)$

\begin{tabular}{|c|c|c|c|c|c|c|c|c|}
\hline \multirow[t]{2}{*}{ Parameter } & \multicolumn{4}{|c|}{ Ipratropium bromide $(500 \mu \mathrm{g}) / \mathrm{salbutamol}(2.5 \mathrm{mg})$} & \multicolumn{4}{|l|}{ Placebo } \\
\hline & Pre-dose & Post-dose & $\Delta ; \%$ & P-value & Pre-dose & Post-dose & $\Delta ; \%$ & $P$-value \\
\hline $\mathrm{FEV}_{1}(\mathrm{~L})$ & $\mathrm{I} .24(0.4 \mathrm{I})$ & $1.49(0.44)$ & $0.25(0.13) ; 20.2$ & $<0.001$ & $1.24(0.42)$ & $1.24(0.42)$ & $0.0(0.1 \mathrm{I}) ; 0$ & 0.999 \\
\hline $\mathrm{FVC}(\mathrm{L})$ & $2.98(0.92)$ & $3.39(0.96)$ & $0.4 \mid(0.32) ; 13.8$ & $<0.001$ & $2.97(0.90)$ & $2.94(0.83)$ & $-0.03(0.28) ; 0$ & 0.585 \\
\hline $\mathrm{FEV} / \mathrm{FVC}(\%)$ & $42.8(11.0)$ & $45.7(13.4)$ & $2.84(7.7) ; 6.6$ & 0.024 & $43.4(14.1)$ & $43.3(13.5)$ & $-0.09(3.41) ; 0$ & 0.865 \\
\hline
\end{tabular}

Notes: Values are mean (SD). $\Delta$, post- minus pre-dose changes.

Abbreviations: $\mathrm{FEV}_{1}$, forced expiratory volume in I second; FVC, forced vital capacity.

dyspnea-IRV ratios was significant only at 14 and 16 steps/ min (Table 4 and Figure S2).

\section{Cardiopulmonary responses during the 3-MST}

The cardiopulmonary responses at the end of each 3-MST are summarized in Table 4. On average, the 3-MSTs were performed at relative exercise intensities of $76 \%-92 \% \dot{\mathrm{V}} \mathrm{O}_{2 \text { peak }}$, $81 \%-92 \% \mathrm{HR}_{\text {peak }}$ and $69 \%-91 \% \dot{V}_{\text {Epeak }}$. Mean IC, IRV and $V_{\mathrm{T}}$ values at the symptom-limited peak of incremental cycle exercise testing (Table 2) were similar to those observed at the end of each 3-MST, particularly at the two highest stepping rates of 20 and 24 steps/min. $\dot{V}_{\mathrm{E}}$ and IC were higher at the end of each 3-MST after treatment with ipratropium bromide/salbutamol compared with placebo. The higher $\dot{V}_{\mathrm{E}}$ with bronchodilation was secondary to increased $V_{\mathrm{T}}$ expansion with bronchodilation compared with placebo, with little to no change in $f$. IRV values were also significantly higher after treatment with ipratropium bromide/salbutamol vs placebo at the end of each 3-MST, except for $20 \mathrm{steps} / \mathrm{min}$.

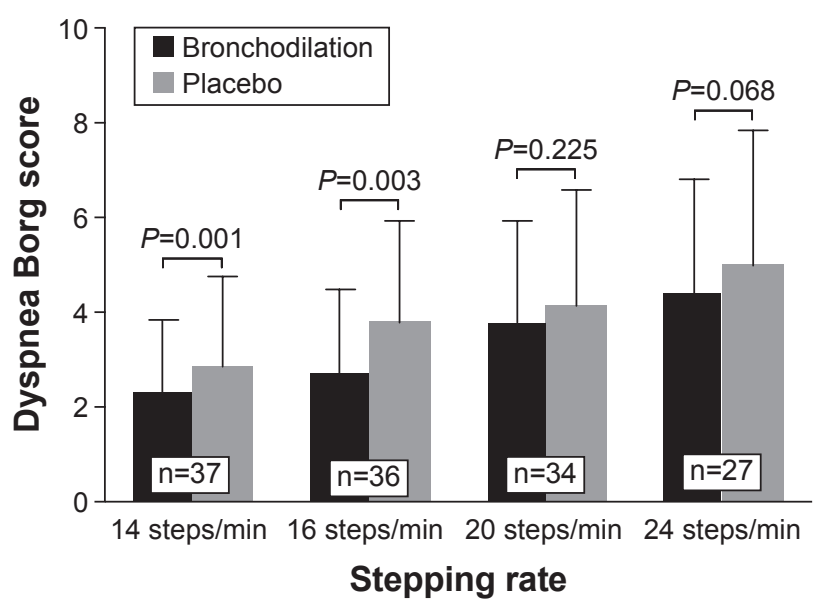

Figure 3 Effect of nebulized fixed-dose combination of ipratropium bromide $(500 \mu \mathrm{g}) / \mathrm{salbutamol}(2.5 \mathrm{mg})\left(\right.$ Combivent $\left.^{\circledR}\right)$ and $0.9 \%$ saline placebo on Borg 0-10 scale intensity ratings of dyspnea at the end of the third minute of exercise.

Note: The number of patients completing all 3 minutes of exercise at 14, 16, 20 and 24 steps/min under both treatment conditions are indicated in the white boxes.
Ipratropium bromide/salbutamol had no demonstrable effect on $\dot{V} \mathrm{O}_{2}, \dot{V} \mathrm{CO}_{2}, \mathrm{HR}$ and $\mathrm{SpO}_{2}$ responses at the end of each 3-MST.

\section{Discussion}

This two-site, randomized, double blind, placebo-controlled, crossover study is the first to demonstrate that the 3-MST, when performed at stepping rates of 14 and 16 steps $/ \mathrm{min}$, is responsive to detect the relief of exertional dyspnea after acute bronchodilation in patients with moderate-tosevere COPD.

Patients in this study had GOLD stage 2-3 COPD and extensive physiological impairment during symptom-limited incremental cardiopulmonary cycle exercise testing as evidenced by dynamic lung hyperinflation $(0.70 \mathrm{~L}$ IC decrease from rest to end exercise), abnormal restrictive constraints on $V_{\mathrm{T}}$ expansion (peak IRV of $0.48 \mathrm{~L}$ ), ventilatory inefficiency (peak $\dot{V}_{\mathrm{E}} / \dot{V} \mathrm{CO}_{2}$ ratio of 36.8) and "severe" to "very severe" dyspnea in the context of a low peak power output $(\approx 56 \%$ of predicted value). The sample population studied herein therefore exhibited classical exercise physiological and symptomatic features of COPD.

Some methodological aspects of this study should be considered for proper interpretation of the data. First, a prerequisite to studying the responsiveness of the 3-MST to bronchodilation is that the active medication significantly improves static and dynamic pulmonary function. The active medication used in the study, ipratropium bromide/ salbutamol, provided considerable bronchodilation at rest and lung deflation during exercise $\left(0.25 \mathrm{~L}\right.$ increase in $\mathrm{FEV}_{1}$ and 0.19-0.29 L increase in IC during exercise vs placebo) that was expected to translate into exertional dyspnea relief. ${ }^{25-27}$ This degree of bronchodilation, obtained in patients who were weaned from their bronchodilators and after the administration of two short-acting bronchodilators, is consistent with the results of large clinical trials involving patients with GOLD stage 2-3 COPD and using similar methodology to estimate the response to bronchodilation. ${ }^{28,29}$ As such, we 


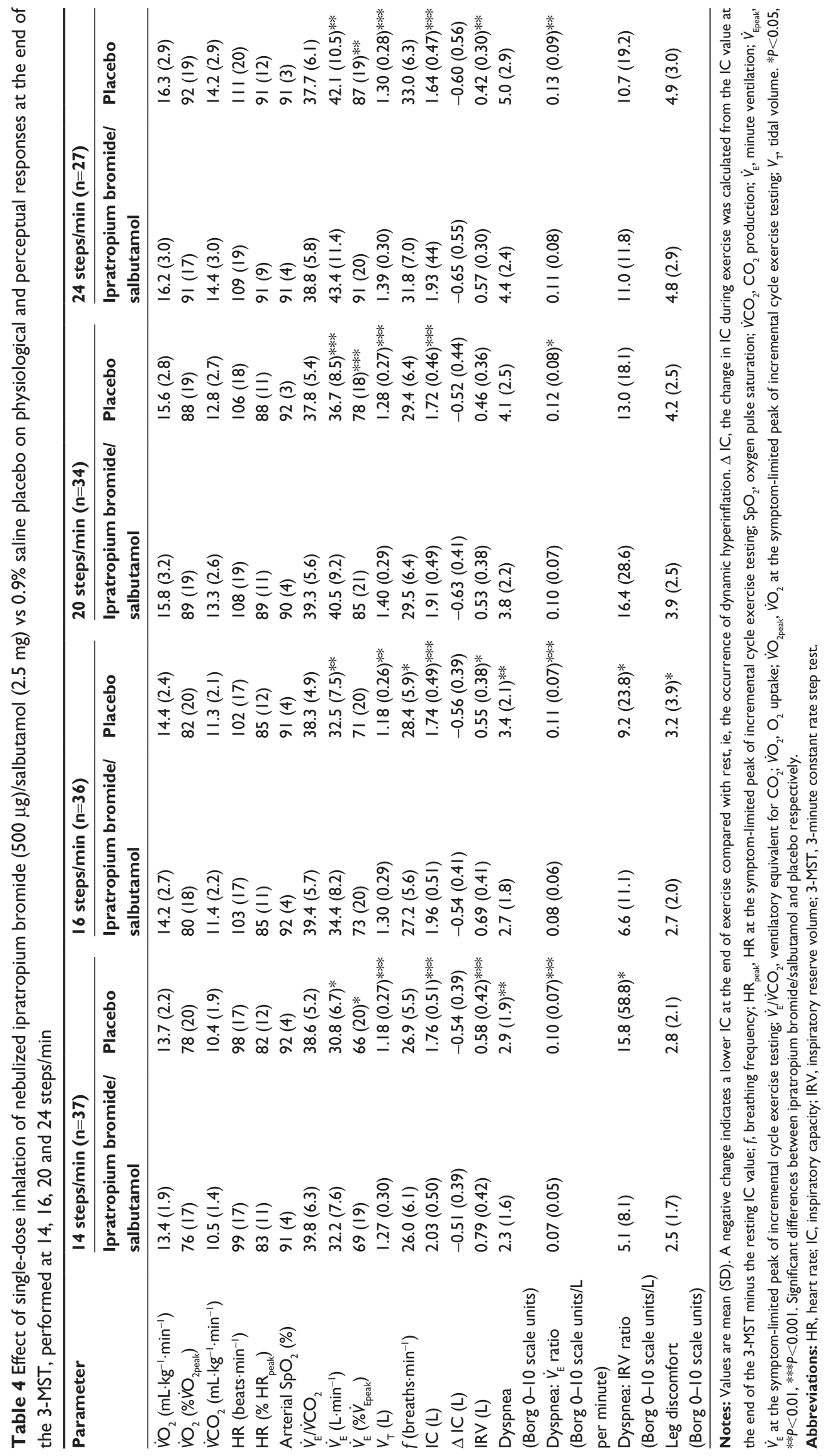


propose that the current study population should be reflective of a broad COPD population. Considering the relatively short bronchodilation time course of the bronchodilators used in the current study, it is possible that the last 3-MST of each study visit was performed in a time window during which the bronchodilation effect was diminishing. This may have contributed to some variability in the results, although randomization of the stepping rate order should have helped minimizing this effect.

The stepping rates were selected based on the results of Perrault et a ${ }^{11,15}$ who reported on the development and testretest reproducibility of the 3-MST performed at stepping rates of 18, 22, 26 and 32 steps/min in patients with GOLD stage 2-4 COPD. In Perrault et al's study, $<70 \%$ of the patients were able to complete all 3 minutes of exercise at both 26 and 32 steps/min, indicating that these stepping rates are likely too intense for routine clinical use in COPD. ${ }^{11}$ Thus, lower stepping rates were used in the current study and we observed, as a result, consistently higher completion rates: $93 \%, 90 \%, 85 \%$ and $68 \%$ for $14,16,20$ and 24 steps $/ \mathrm{min}$, respectively. The choice of Borg 0-10 scale to assess dyspnea was based on its wide use in COPD research and for consistency purpose with our research program on the use of the 3-minute walking and stepping tests. ${ }^{11,15}$ One interesting feature of the Borg scale is that we have a sense of how to interpret the magnitude of changes seen with treatment, a 1-point decrease in Borg dyspnea score being considered clinically relevant. ${ }^{24} \mathrm{We}$ did not consider the presence of chronic dyspnea during daily activities or the achievement of a certain threshold of Borg dyspnea score during the 3-MST as inclusion criteria for this study. In future investigations, it would seem relevant to determine whether the responsiveness of the 3-MST could differ according to the presence/absence of chronic dyspnea during daily activities or during exertion.

Statistically significant relief of patient-perceived dyspnea was observed at the end of the 3-MST performed at the two lowest stepping rates of 14 and 16 steps/min after treatment with ipratropium bromide/salbutamol vs placebo. The magnitude of dyspnea relief following bronchodilation at these step rates was, on average, 0.6-0.7 Borg scale units. Moreover, although the range of symptom relief was variable, dyspnea intensity ratings were reduced by $\geq 1$ Borg scale unit (the minimal clinically important difference for this variable ${ }^{24}$ ) in $45 \%$ of our patients during stepping at 14 steps/min (by $1.3 \pm 0.8$ Borg scale units) and 16 steps/min (by $1.6 \pm 1.0$ Borg scale units) after treatment with ipratropium bromide/ salbutamol vs placebo (Figures 3 and S1). As reviewed in detail elsewhere, ${ }^{30}$ exertional dyspnea relief following bronchodilation in the current study likely reflected the established benefits of reduced dynamic operating lung volumes (ie, increased IC and IRV, respectively, reflecting reduced end-expiratory and end-inspiratory lung volumes) and increased $V_{\mathrm{T}}$ expansion on neuromechanical coupling of the respiratory system in COPD. ${ }^{26,31,32}$

In contrast to the abovementioned details, single-dose inhalation of ipratropium bromide/salbutamol had no significant effect on ratings of perceived dyspnea during exercise at 20 and $24 \mathrm{steps} / \mathrm{min}$. The inability of the two higher stepping rates to demonstrate reductions in dyspnea could be related to the higher exercise intensity and ventilatory requirements at which these tests were conducted. Emerging evidence suggests that patients with COPD and interstitial pulmonary disease ${ }^{31,33}$ experience intolerable dyspnea once their dynamic IRV decreases to a critical minimal value of $\sim 0.60 \mathrm{~L}$ below the total lung capacity during exercise (recently termed the "O'Donnell threshold"34). Considering the relatively high levels of $\dot{V}_{\mathrm{E}}$ reached while stepping at 20 and 24 steps $/$ min (ie, $85 \%-91 \% \dot{V}_{\text {Epeak }}$ ), patients were most likely breathing on the uppermost (alinear) extreme of the respiratory system's sigmoid pressure-volume relationship, where modest increases in dynamic IRV to levels that remained below the O'Donnell threshold of $\sim 0.60 \mathrm{~L}$ after treatment with ipratropium bromide/salbutamol (from 0.46 to $0.53 \mathrm{~L}$ at $20 \mathrm{steps} / \mathrm{min}$ and from 0.42 to $0.57 \mathrm{~L}$ at $24 \mathrm{steps} / \mathrm{min}$ ) would not be expected to translate into exertional dyspnea relief despite significant increases in IC and $V_{\mathrm{T}}$ expansion. The corollary of this is that the relief of dyspnea during exercise at 14 and 16 steps/min after bronchodilation was associated with physiologically important increases in dynamic IRV from below to above the O'Donnell threshold, ie, from 0.58 to $0.79 \mathrm{~L}$ at $14 \mathrm{steps} / \mathrm{min}$ and from 0.55 to $0.69 \mathrm{~L}$ at $16 \mathrm{steps} / \mathrm{min}$. Apart from this physiological explanation, loss in statistical power due to a lower number of patients completing the $24 \mathrm{steps} / \mathrm{min}$ rate could have contributed to the loss of statistical significance in the reduction of dyspnea at this stepping rate.

The smaller number of patients who were able to complete the two highest stepping rates raises the issue of how to select the optimal stepping rate for use in COPD. For example, we could be targeting a standard stepping rate across patients, relative to the severity of the disease. In this context, the 3-MST performed at 14 or 16 steps/ min would seem appropriate choices in most patients with GOLD stage 2-3 disease, where these stepping rates induce levels of dyspnea that are sufficiently intense to be amenable 
to therapy. Alternatively, one might want to target a standard "stimulus" by tailoring the stepping rates to the individual patient's characteristics (age, sex, body mass index, \% predicted $\mathrm{FEV}_{1}$, etc.) so as to elicit a standardized level of exertional dyspnea across patients with a wide range of disease severity. Further investigation is necessary to compare these two possible approaches.

\section{Conclusion}

This randomized, controlled, and multi-center study demonstrated that the 3-MST, when performed at stepping rates of 14 and 16 steps/min, was able to detect statistically significant and potentially clinically meaningful relief of exertional dyspnea after acute bronchodilation in patients with moderateto-severe COPD. Further considerations are required to extend the use of the 3-MST to a broader patient population, including patients with mild COPD. Finally, the results of the current study provide a scientific rationale for the use of the 3-MST protocol to detect the relief of physical activityrelated dyspnea in the context of clinical (therapeutic) trials in COPD and, perhaps, also in other chronic pulmonary disorders such as interstitial pulmonary fibrosis.

\section{Acknowledgments}

The authors would like to thank Marthe Bélanger, MarieJosée Breton, Brigitte Jean, Josée Picard and Jacinthe Baril in accomplishing this study. They also thank Julie Milot, Yves Lacasse, Steeve Provencher, Pierre Leblanc, Eric Nadreau, Alisha Maini and Sara Abdallah for their help with the exercise tests. Dennis Jensen was supported by a Chercheurs-boursiers Junior 1 salary award from the Fonds de la Recherche Québec-Santé and by a William Dawson Research Scholars Award (McGill University). François Maltais holds a GlaxoSmithKline (GSK)/Canadian Institute of Health Research (CIHR) Research Chair on COPD at Université Laval. This work was supported by an unrestricted and investigator-initiated grant from Boehringer Ingelheim (Canada) Limited to François Maltais. The sponsor of the study had no role in study design, data collection and interpretation, preparation of the manuscript or decision to submit the manuscript for publication. The abstract of this paper was presented at the 2014 American Thoracic Society Conference (San Diego, CA, USA) as a poster presentation with interim findings. The poster's abstract was published in "Poster Abstracts" in the American Journal of Respiratory and Critical Care Medicine (ATS International Conference Abstracts Issue): http://www.atsjournals.org/doi/abs/10.1164/ ajrccm-conference.2014.189.1_MeetingAbstracts.A5862.
However, the current paper has never been published and is not submitted for publication elsewhere at this time.

\section{Author contributions}

Conception and design: BB, FM and DJ. Data collection and analysis: BB and CAW-M. Drafting and editing of the manuscript for important intellectual content: BB, CAW-M, JB, HP, DJ and FM. AH, an employee of the sponsor, has been involved in the scientific development of this InvestigatorInitiated Research Program. ${ }^{11,15}$ AH reviewed and commented on the manuscript with no obligation for the academic authors to incorporate any proposed changes. All authors contributed toward data analysis, drafting and critically revising the paper and agree to be accountable for all aspects of the work.

\section{Disclosure}

JB reports unrestricted educational grants from GSK, Pfizer, Boehringer Ingelheim (Canada) Limited and AstraZeneca, and funding for the operations of CanCOLD Longitudinal Epidemiological Study from the CIHR/Rx\&D Collaborative Research Program with industry partners such as AstraZeneca Canada, Boehringer Ingelheim (Canada) Limited, GSK Canada, Merck and Novartis. DJ has received an unrestricted research grant from Boehringer Ingelheim (Canada) Limited and an education grant from AstraZeneca Canada. FM received fees for speaking at conferences sponsored by Boehringer Ingelheim (Canada) Limited, GSK, Novartis and Grifols; research grants for participating in multicenter trials sponsored by GSK, Boehringer Ingelheim (Canada) Limited, Astra Zeneca, Nycomed and Novartis and unrestricted research grants from Boehringer Ingelheim (Canada) Limited and GSK.BB, CAW-M, AH and HP report no conflicts of interest in this work.

\section{References}

1. Celli BR, MacNee W; ATS/ERS Task Force. Standards for the diagnosis and treatment of patients with COPD: a summary of the ATS/ERS position paper. Eur Respir J. 2004;23(6):932-946.

2. O'Donnell DE, Aaron S, Bourbeau J, et al. Canadian Thoracic Society recommendations for management of chronic obstructive pulmonary disease - 2007 update. Can Respir J. 2007;14(suppl B):5B-32B.

3. Vestbo J, Hurd SS, Agusti AG, et al. Global strategy for the diagnosis, management, and prevention of chronic obstructive pulmonary disease: GOLD executive summary. Am J Respir Crit Care Med. 2013;187(4): 347-365

4. Borel B, Provencher S, Saey D, Maltais F. Responsiveness of various exercise-testing protocols to therapeutic interventions in COPD. Pulm Med. 2013;2013:410748.

5. Brouillard C, Pepin V, Milot J, Lacasse Y, Maltais F. Endurance shuttle walking test: responsiveness to salmeterol in COPD. Eur Respir J. 2008; 31(3):579-584.

6. O'Donnell DE, Fluge T, Gerken F, et al. Effects of tiotropium on lung hyperinflation, dyspnoea and exercise tolerance in COPD. Eur Respir J. 2004;23(6):832-840. 
7. O'Donnell DE, Voduc N, Fitzpatrick M, Webb KA. Effect of salmeterol on the ventilatory response to exercise in chronic obstructive pulmonary disease. Eur Respir J. 2004;24(1):86-94.

8. Maltais F, Hamilton A, Marciniuk D, et al. Improvements in symptomlimited exercise performance over $8 \mathrm{~h}$ with once-daily tiotropium in patients with COPD. Chest. 2005;128(3):1168-1178.

9. Casaburi R, Maltais F, Porszasz J, et al. Effects of tiotropium on hyperinflation and treadmill exercise tolerance in mild to moderate chronic obstructive pulmonary disease. Ann Am Thorac Soc. 2014;11(9): 1351-1361.

10. Zhang X, Waterman LA, Ward J, Baird JC, Mahler DA. Advantages of endurance treadmill walking compared with cycling to assess bronchodilator therapy. Chest. 2010;137(6):1354-1361.

11. Perrault H, Baril J, Henophy S, Rycroft A, Bourbeau J, Maltais F. Paced-walk and step tests to assess exertional dyspnea in COPD. COPD. 2009;6(5):330-339.

12. Lahaije AJ, van Helvoort HA, Dekhuijzen PN, Heijdra YF. Physiologic limitations during daily life activities in COPD patients. Respir Med. 2010;104(8):1152-1159.

13. Vaes AW, Wouters EF, Franssen FM, et al. Task-related oxygen uptake during domestic activities of daily life in patients with COPD and healthy elderly subjects. Chest. 2011;140(4):970-979.

14. Castro AA, Porto EF, Iamonti VC, de Souza GF, Nascimento OA, Jardim JR. Oxygen and ventilatory output during several activities of daily living performed by COPD patients stratified according to disease severity. PLoS One. 2013;8(11):e79727.

15. Sava F, Perrault H, Brouillard C, et al. Detecting improvements in dyspnea in COPD using a three-minute constant rate shuttle walking protocol. COPD. 2012;9(4):395-400.

16. Combivent Inhalation Aerosol Study Group. In chronic obstructive pulmonary disease, a combination of ipratropium and albuterol is more effective than either agent alone. An 85-day multicenter trial. COMBIVENT Inhalation Aerosol Study Group. Chest. 1994;105(5): 1411-1419.

17. American Thoracic Society. Standards for the diagnosis and care of patients with chronic obstructive pulmonary disease. American Thoracic Society. Am J Respir Crit Care Med. 1995;152(5 pt 2):S77-S121.

18. Quanjer PH, Tammeling GJ, Cotes JE, Pedersen OF, Peslin R, Yernault JC. Lung volumes and forced ventilatory flows. Report Working Party Standardization of Lung Function Tests, European Community for Steel and Coal. Official Statement of the European Respiratory Society. Eur Respir J Suppl. 1993;16:5-40.

19. Hankinson JL, Odencrantz JR, Fedan KB. Spirometric reference values from a sample of the general U.S. population. Am J Respir Crit Care Med. 1999;159(1):179-187.
20. Gandevia B, Hugh-Jones P. Terminology for measurements of ventilatory capacity; a report to the thoracic society. Thorax. 1957;12(4): 290-293.

21. O'Donnell DE, Revill SM, Webb KA. Dynamic hyperinflation and exercise intolerance in chronic obstructive pulmonary disease. Am J Respir Crit Care Med. 2001;164(5):770-777.

22. Borg GA. Psychophysical bases of perceived exertion. Med Sci Sports Exerc. 1982;14(5):377-381

23. American Thoracic Society; American College of Chest Physicians. ATS/ACCP statement on cardiopulmonary exercise testing. Am J Respir Crit Care Med. 2003;167(2):211-277.

24. Ries AL. Minimally clinically important difference for the UCSD Shortness of Breath Questionnaire, Borg Scale, and Visual Analog Scale. COPD. 2005;2(1):105-110.

25. O'Donnell DE, Lam M, Webb KA. Measurement of symptoms, lung hyperinflation, and endurance during exercise in chronic obstructive pulmonary disease. Am J Respir Crit Care Med. 1998;158(5 pt 1): $1557-1565$.

26. O'Donnell DE, Lam M, Webb KA. Spirometric correlates of improvement in exercise performance after anticholinergic therapy in chronic obstructive pulmonary disease. Am J Respir Crit Care Med. 1999; 160(2):542-549.

27. Pepin V, Saey D, Whittom F, LeBlanc P, Maltais F. Walking versus cycling: sensitivity to bronchodilation in chronic obstructive pulmonary disease. Am J Respir Crit Care Med. 2005;172(12):1517-1522.

28. Tashkin DP, Celli B, Decramer M, et al. Bronchodilator responsiveness in patients with COPD. Eur Respir J. 2008;31(4):742-750.

29. Wedzicha JA, Banerji D, Chapman KR, et al. Indacaterol-glycopyrronium versus salmeterol-fluticasone for COPD. N Engl J Med. 2016;374(23): 2222-2234.

30. O'Donnell DE, Banzett RB, Carrieri-Kohlman V, et al. Pathophysiology of dyspnea in chronic obstructive pulmonary disease: a roundtable. Proc Am Thorac Soc. 2007;4(2):145-168.

31. O'Donnell DE, Hamilton AL, Webb KA. Sensory-mechanical relationships during high-intensity, constant-work-rate exercise in COPD. J Appl Physiol. 2006;101(4):1025-1035.

32. Belman MJ, Botnick WC, Shin JW. Inhaled bronchodilators reduce dynamic hyperinflation during exercise in patients with chronic obstructive pulmonary disease. Am J Respir Crit Care Med. 1996;153(3):967-975.

33. Faisal A, Alghamdi BJ, Ciavaglia CE, et al. Common mechanisms of dyspnea in chronic interstitial and obstructive lung disorders. Am J Respir Crit Care Med. 2016;193(3):299-309.

34. Casaburi R, Rennard SI. Exercise limitation in chronic obstructive pulmonary disease. The O'Donnell threshold. Am J Respir Crit Care Med. 2015;191(8):873-875.
International Journal of COPD

\section{Publish your work in this journal}

The International Journal of COPD is an international, peer-reviewed journal of therapeutics and pharmacology focusing on concise rapid reporting of clinical studies and reviews in COPD. Special focus is given to the pathophysiological processes underlying the disease, intervention programs, patient focused education, and self management protocols.

\section{Dovepress}

This journal is indexed on PubMed Central, MedLine and CAS. The manuscript management system is completely online and includes a very quick and fair peer-review system, which is all easy to use. Visit http://www.dovepress.com/testimonials.php to read real quotes from published authors. 